\title{
Analysis of Urban Change in Shenzhen City Based on Landsat Archived Data
}

\author{
Tingting Chen, Hermann Josef Kaufmann \\ Navigation and Remote Sensing Group, Institute of Space Science, Shandong University, Weihai, China \\ Email: xiazheteng1129@126.com, kofmich@yahoo.com
}

How to cite this paper: Chen, T.T. and Kaufmann, H.J. (2018) Analysis of Urban Change in Shenzhen City Based on Landsat Archived Data. Journal of Computer and Communications, 6, 146-154. https://doi.org/10.4236/jcc.2018.611014

Received: October 28, 2018

Accepted: November 12, 2018 Published: November 19, 2018

\begin{abstract}
With the ongoing development of economy and urbanization in China, the change of land use types has attracted more and more attention. In this study we focused on the urban development of Shenzhen City, Guangdong Province, analyzing Landsat $5 \mathrm{TM}$ and Landsat 8 OLI data. We used an SVM based classification, a land transfer matrix approach, a directional growth analysis method and we calculated the inversion of land surface temperature to derive information of land cover changes that occurred in the time period between 1987 and 2017. The results are combined with Shenzhen's economy, transportation policy and other aspects to find the driving forces of the urban development. The results show that during the observed 30 years, the area of construction land has increased significantly. Most of it is converted from other lands, and some of them are reclaimed. Most rapidly developing are areas west and northwest of the Bao'an, Nanshan and Longhua. The vegetated areas decreased slightly. Caused by the continuous increase of the construction land, the so-called heat island effect emerges slightly but continuously.
\end{abstract}

\section{Keywords}

Change Detection, Urbanization, Heat Island, Multitemporal Analysis Landsat Data

\section{Introduction}

Since the reform and opening up, China's urbanization is developing rapidly. Land use research has become a hot issue discussed by scholars at home and abroad. In order to further know the development law of urbanization in China and solve some problems brought about by the rapid development of urbanization, remote sensing technology is used to study Shenzhen from different directions, such as urban expansion, driving force and influence. 


\section{Survey of Research Area}

The city of Shenzhen is adjacent to Hong Kong and located in the south of the Guangdong Province. Its coordinates are as follows: east longitude $113^{\circ} 46^{\prime}$ $114^{\circ} 37^{\prime}$; north latitude $22^{\circ} 27^{\prime}-22^{\circ} 52^{\prime}$. Shenzhen is characterized by a subtropical monsoon climate with pleasant wind and rich precipitation. The city has 6 administrative districts and 3 new districts (Figure 1), with a total area of 1997.27 square kilometers. By the end of 2017, the resident population of Shenzhen summed up to $12,528,300$.

Shenzhen is the first special economic zone set up in China. It has developed from a small fisherman's village to a big city of international flavor in just 30 years. Its per capita GDP (Gross Domestic Product) ranks first in China, creating a miracle of urbanization, industrialization and modernization in the world.

\section{Materials and Methods}

\subsection{Data Sources}

The data sources used in this paper are archived multi-temporal Landsat data that are widely used in the field of remote sensing of Earth resources and environment [1]. The Landsat 5 TM data where recorded before 2012 and the Landsat 8 OLI data taken after 2013. Images were selected corresponding to the autumn time and a minimum of cloud cover.

\subsection{Data Preprocessing}

In a first step, ENVI commercial software is used to pre-process the original imagery, such as subset generation, radiometric calibration, atmospheric correction using flash, image registration and seamless mosaicing. According to the band characteristics of TM and OLI images, altogether 345 and 764 bands are combined to fully enhance the spectral content of all images.

The study area is divided into four categories, namely construction land, vegetated areas, waterbodies, and other lands and consecutively classified by a

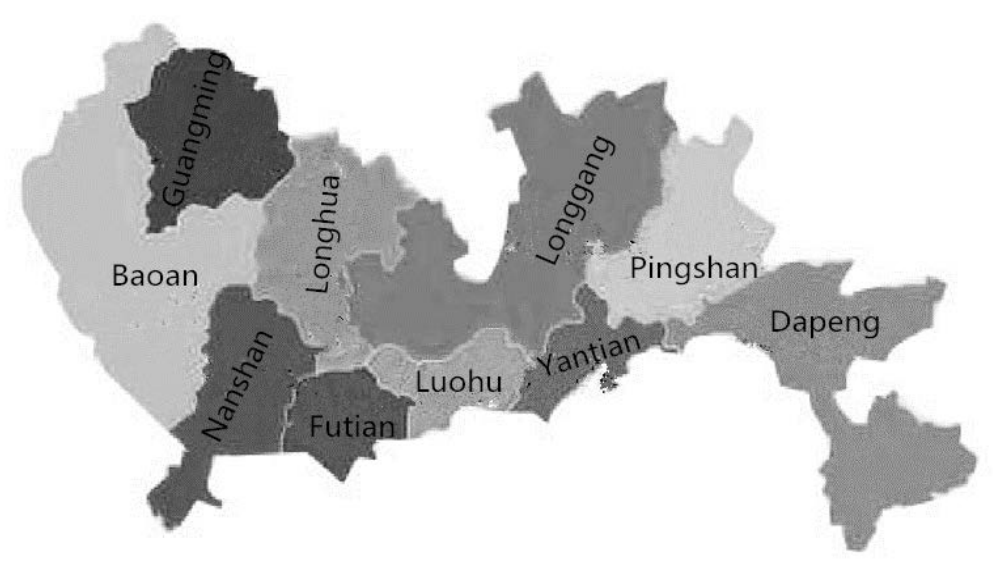

Figure 1. Shenzhen administrative districts. 
supervised approach, the Support Vector Machine (SVM). This is a data mining method based on statistical learning theory, which can deal with classification, recognition and time series analysis [2]. The accuracy of the method was evaluated by using a confusion matrix. The overall accuracy was $96.3850 \%$, and the Kappa coefficient resulted to 0.8919 . The land use in the study area was monitored dynamically and the land change characteristics [3] were obtained.

\section{Results and Analysis}

\subsection{Land Use Analysis in Shenzhen in Recent 30 Years}

\subsubsection{Amplitude of Variation}

The area and percentage of different land types in different years were calculated using the ENVI, and the land transfer matrix was calculated based on the results of the land classification approach.

From Figure 2, it can be deduced that water bodies and vegetated areas are the most prominent categories. Vegetated areas decreased significantly while the water bodies decreased just slightly in the past 30 years. They changed from $45 \%$ and $33 \%$ to $36 \%$ and $30 \%$. Whereas the areas are reduced, the changes are visibly not obvious. Caused by urbanization processes, the category other lands began to decrease, from $15 \%$ in 1987 to $4 \%$ in 2017 . The area of construction land increased from $7 \%$ in 1987 to $30 \%$ in 2017 by more than a factor of 4 . From Figure 3 we learn that the category construction land tends to increase in the future and will expand in all directions.

According to the results given by the land transfer matrix in Table 1 ( + is increasing, - is decreasing), the four types of land use are transformed with each

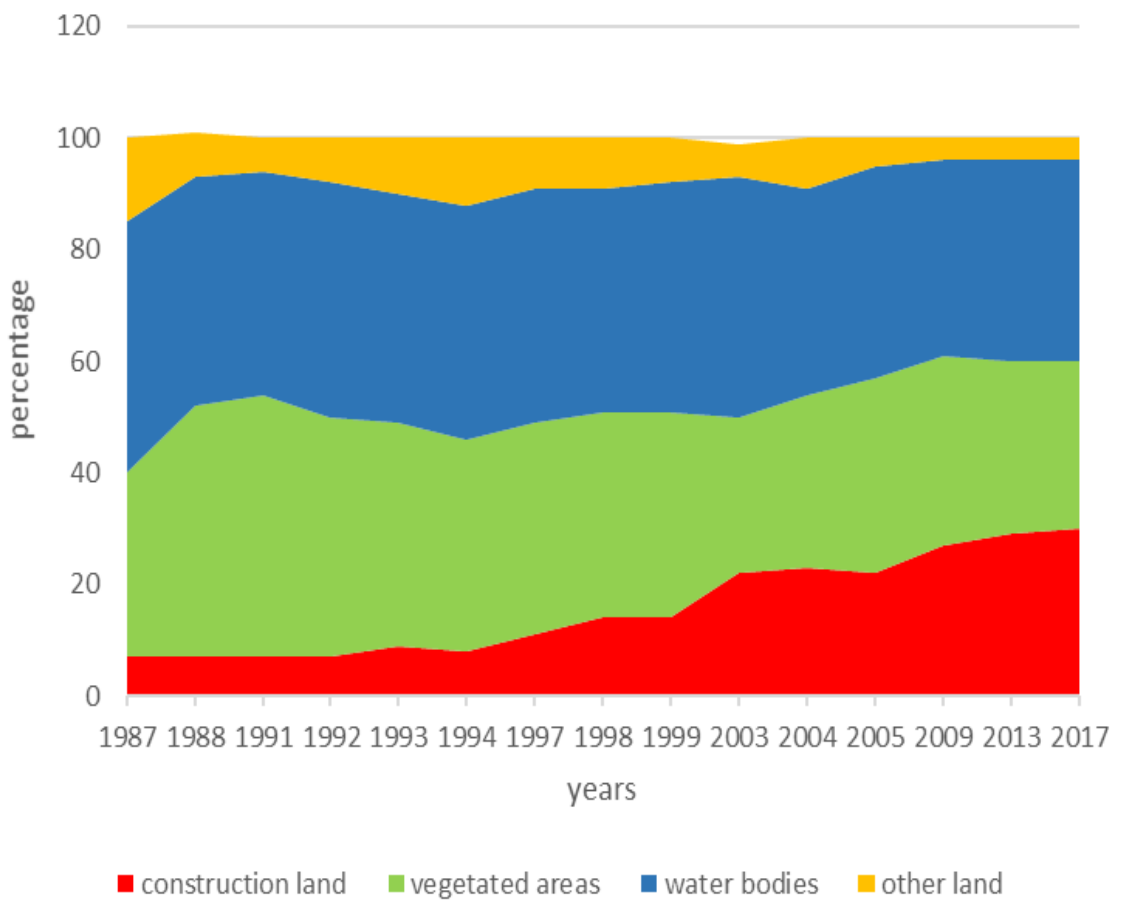

Figure 2. Percentage of land use types during the time period from 1987-2017. 


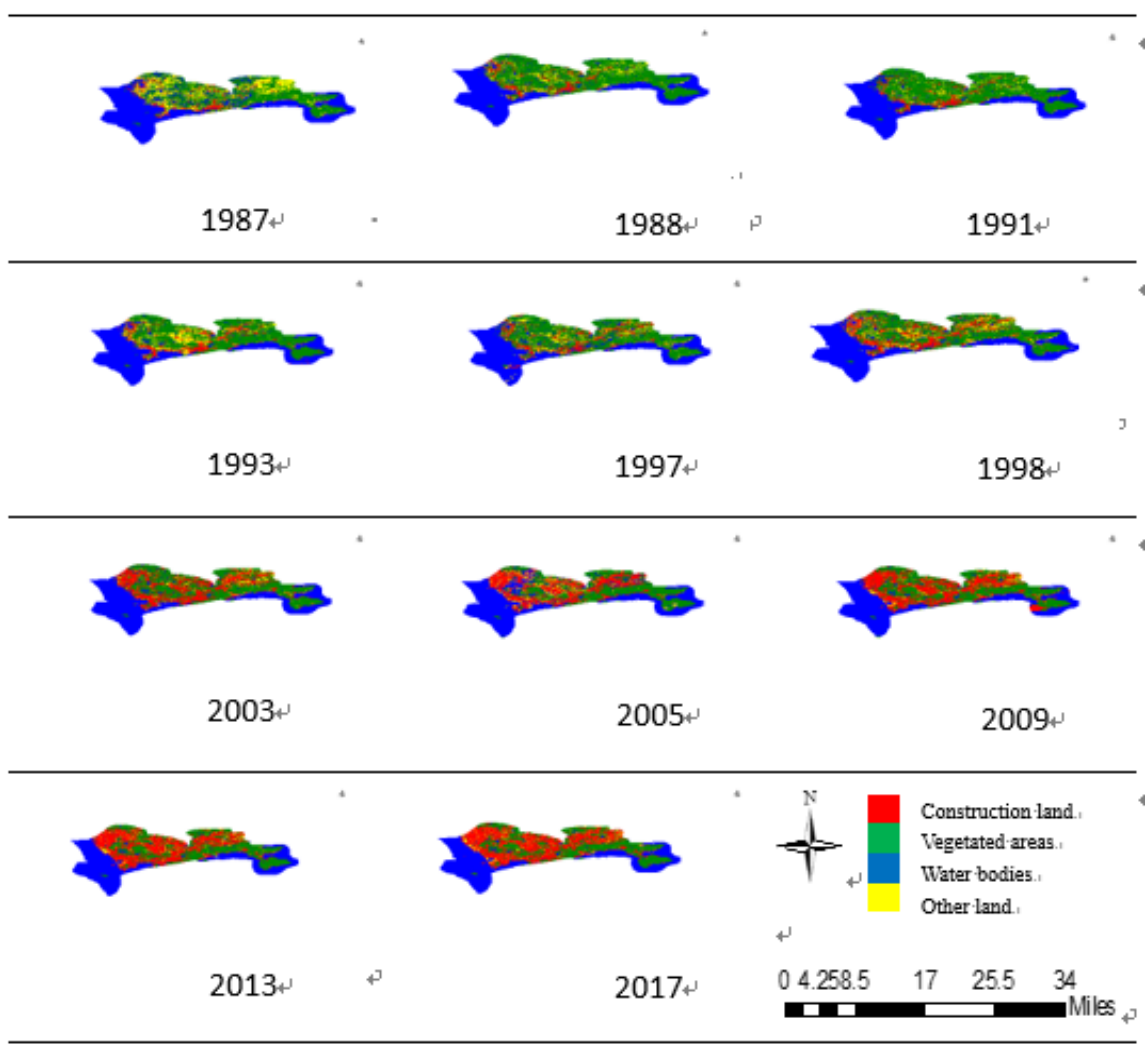

Figure 3. Temporal and spatial variations of different land use types.

Table 1. Land transfer matrix.

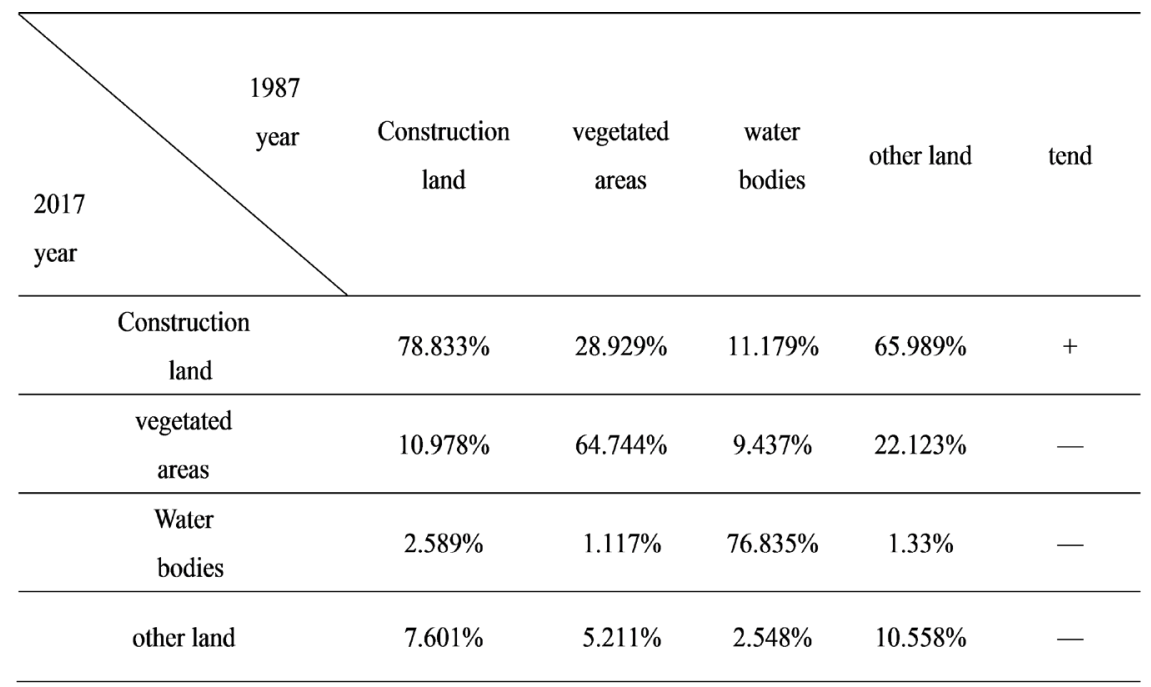

other. $66 \%$ of the other land types are converted to construction land, while $8 \%$ of the construction land is converted to other land use types. The total amount of vegetation and water and their transfer is basically unchanged. The ecological evolution is positive, which also reflects Shenzhen's self-reliance and the protection of the ecosystem. In general, the other land use types are the biggest transferors and the construction land is the largest recipient, indicating that in recent 
years, with the continuous upgrading of the economy, the demand for urban construction land increased.

\subsubsection{Direction of Change}

The classification grid map was transformed into a vector map based on ENVI and used as input to ArcGIS software. ArcGIS was used to draw an eight-direction circle and to fuse it with each land type to see their respective expansion in the distinct directions.

Figure 4 indicates that the construction land in Shenzhen has principally expanded in all directions. The western region developed much faster than the eastern region, namely the Baoan District, the Nanshan District, and the Futian District. This may be related to its geographical location. The western part of Shenzhen is located near the Pearl River Estuary, known as the Pearl River Delta economic zone, where the economic development is faster than at the remaining vicinity of Shenzhen [4].

\subsection{Analysis of Land Surface Temperature in Shenzhen in September 2016}

An important information source for the urban surface temperature is the thermal band 10 of the Landsat 8 OLI sensor. The necessary atmospheric correction method has been adopted. The total amount of thermal radiation observed by the satellite sensor subtracts the estimated atmospheric influence on the surface thermal radiation. The resulting surface thermal radiation intensity is then converted into the corresponding surface temperature (Figure 5).

$B\left(T_{\lambda}\right)$ is the total amount of thermal radiation observed by the satellite sensor. It consists of three parts (Equation (1)). The upward radiance of the atmosphere ( $B \uparrow$ ), the energy of the downward radiation of the atmosphere reflected by the Earth and the energy of the Earth's true radiance reaching the satellite sensor

$$
B\left(T_{\lambda}\right)=\left[e B\left(T_{s}\right)+(1-e) B \downarrow\right] \tau+B \uparrow
$$

Equation (2) shows the calculation of the blackbody radiance (temperature T) based on the thermal infrared band:

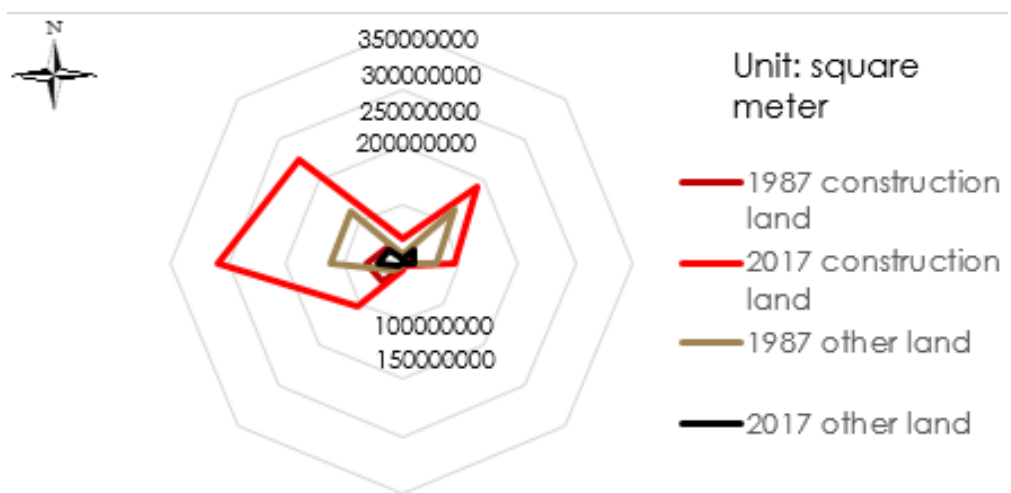

Figure 4. Eight directions of land use. 


$$
B\left(T_{s}\right)=\left[B\left(T_{\lambda}\right)-B \uparrow-\tau(1-e) B \downarrow\right] / \tau e
$$

The surface temperature $\left(T_{S}\right)$ is given by Equation (3):

$$
T_{S}=K_{2} / \operatorname{In}\left(K_{1} / B\left(T_{S}\right)+1\right)
$$

where

$B \downarrow=$ atmospheric downward radiance, $e=$ surface emissivity, $\tau=$ atmospheric transmittance in the thermal infrared band.

$K_{1}, K_{2}$ be obtained from the metadata file. Two equations calculating the specific steps. The atmospheric profile parameters are provided by the following web site: http://atmcorr.gsfc.nasa.gov/):

As shown in Figure 6, the temperatures of the urban built-up areas are much higher than that of the marginal area and water bodies. The mean temperature of the study area accounts for the majority, indicating the existence of a heat island effect in Shenzhen. The temperatures in the central, western, northwestern and

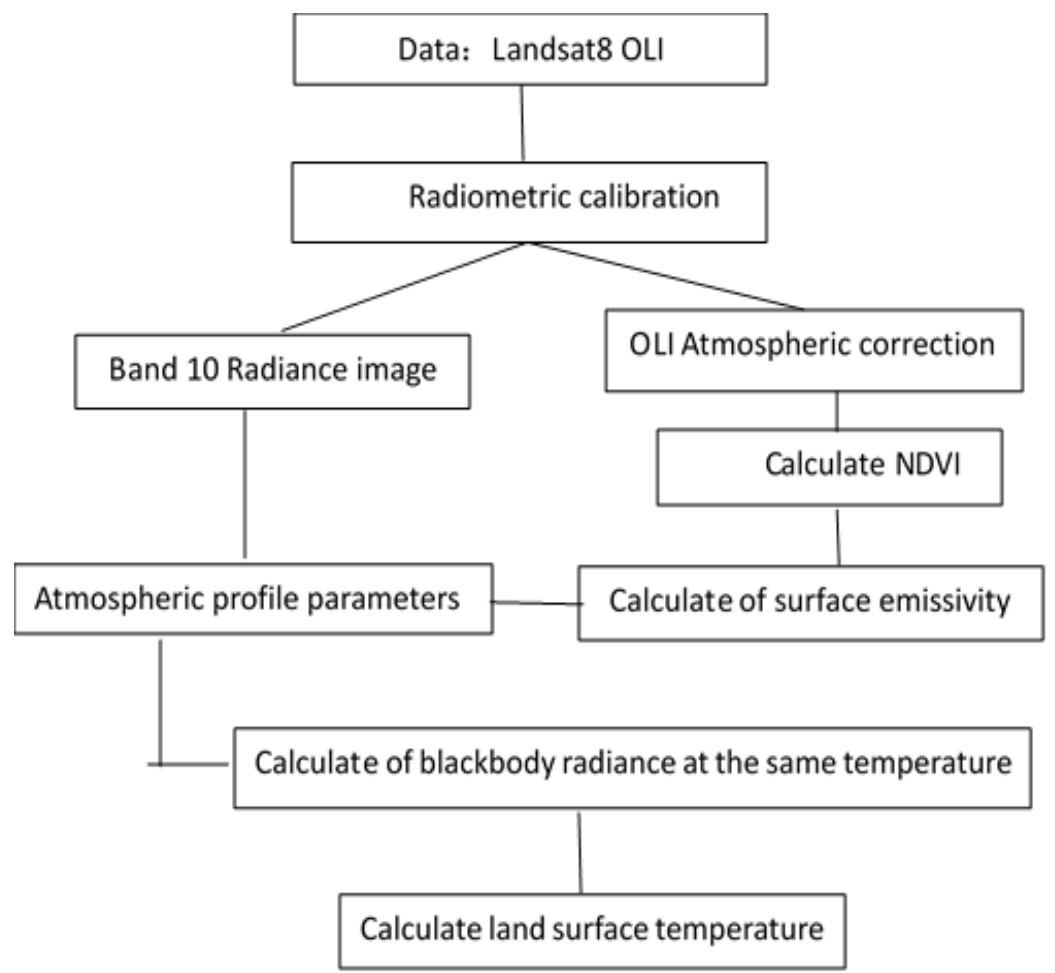

Figure 5. Flow Chart of processing thermal data.

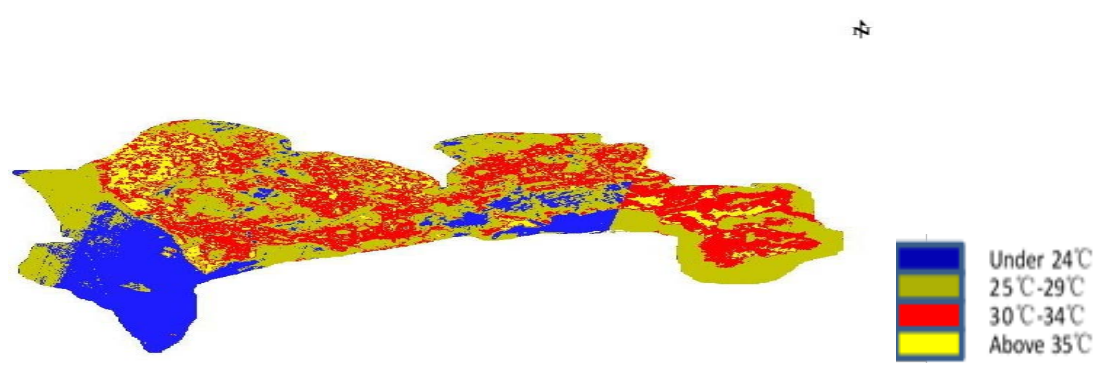

Figure 6. Urban land surface temperatures in September 2016. 
southwestern parts of Shenzhen are higher than 35 degrees, mainly distributed in the Bao'an, the Nanshan and the Futian districts. Concurrently, there is an obvious urban expansion in these districts. There are also some high temperature phenomenon in the Yantian District to the south, in the Longgang District to the northeast and in the Dapeng New District to the southeast. The results of the meteorological data analysis are basically comparable [4]. Most of the low temperature zones are related to the water areas. Shenzhen has not exceeded 37 degrees, accounting for the majority of the 24 to 32 degrees, indicating that the intensity of urban heat island is not intense. This may be related to Shenzhen's geographical location and its development mode. It is surrounded by the sea on three sides. Thus, the water specific, higher heat capacity plays a significant role in regulating the heat island effect [5], the biological production process, and the spatial agglomeration. The degree, scale and types of urban green space are the major compartments that affect the distribution of land surface temperature ([6] [7]). In addition, the spatial distribution of Shenzhen has the character of a multi-center cluster development. The difference between the city and the suburbs is relatively small, and so the urban heat island effect is weak.

\subsection{Analysis of the Driving Force of Land Use Change}

\subsubsection{Economic Factors}

Economic growth is closely related to urbanization and the GDP growth is the most fundamental factor in the expansion of urban land use [8]. Since the reform and opening up, Shenzhen's economy has been developing rapidly. Figure 7 displays the relation between construction areas and the GDP. The value of $\mathrm{R}^{2}$ fitting of 0.8 indicates the correlation between them.

\subsubsection{Traffic Factors}

In the recent thirty years, the infrastructure Shenzhen developed rapidly. The construction of 16 subway lines as long as 189.4 kilometers, newly developed highways, high-speed rail, waterways and other urban infrastructure provided the pathways for the fast development and sprawl of urbanization.

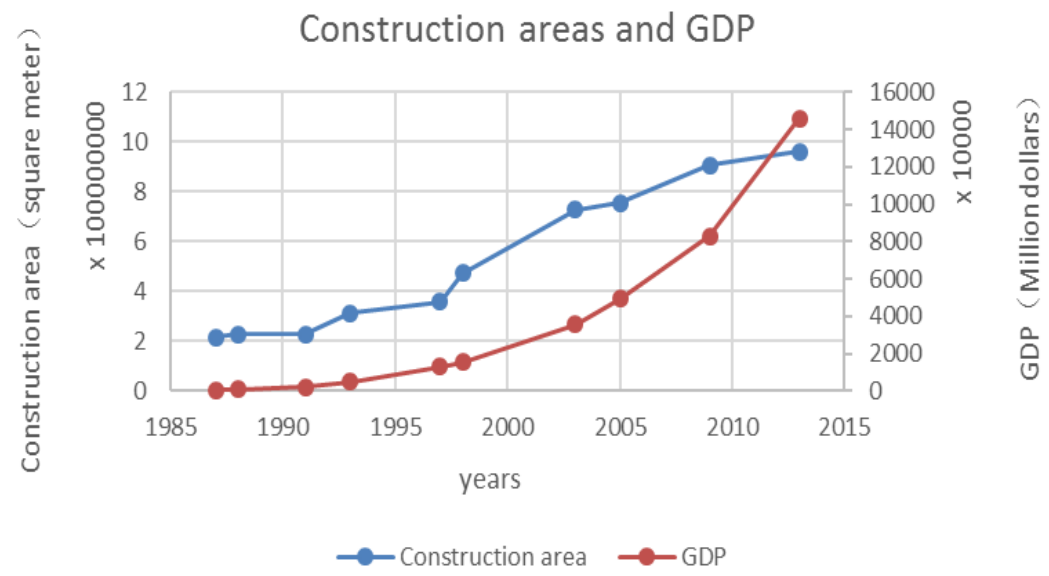

Figure 7. Development of construction areas and GDP. 


\subsubsection{Policy Factors}

Land use change is also affected by policies, that influences the land use structure in different way [9]. Shenzhen is a special economic zone, with many economic policies such as the "three to one subsidy, Shenzhen's $13^{\text {th }}$ Five-Year Plan Outline" that has a great impact on the development of construction land.

\section{Summary of Results}

In this study, the different accomplished methods of analysis based on achieved multi-temporal Landsat satellite data revealed a number of significant results concerning the urbanization and land transfer processes in Shenzhen City and its vicinity.

1) Between the years 1978 and 2017, the types of land use changed significantly. The results show an increase of the construction land, and a decrease of all other classified categories with vegetated areas and other lands more significantly than the water bodies. All types of land use categories are mutual converted.

2) The construction land has expanded into all directions, mainly to the west and northwest. Taking Shenzhen's heart of the City as the center, the eight-directional change map shows the construction land is more concentrated in the Baoan, the Nanshan, the Futian and the Luohu Districts.

3) Land use change is not only triggered by economic development, policies and infrastructure but also influenced by scientific and technological development, and other factors [10].

\section{Acknowledgements}

This paper was completed under the careful guidance of Professor Hermann Josef Kaufmann, who was meticulous and diligent in his studies. He taught students earnestly and helped me a lot. At the same time, I also want to thank Professor Guochang $\mathrm{Xu}$ for his teaching, his profound academic attainments, noble personality, I can benefit greatly from the road to study. Besides, thanks Professor TianheXu, Rang Kang, Shanyu Zhou for their help and support. Finally, I would like to say thank you to all those who care and support me.

\section{Conflicts of Interest}

The authors declare no conflicts of interest regarding the publication of this paper.

\section{References}

[1] Xu, W.F. and Chen, M. (2018) Study on Land Use Change Detection and Driving Force in Beijing Based on Landsat Image. Beijing Surveying and Mapping, 1, 58-61.

[2] Zeng, C., Shen, H. and Zhang, L. (2013) Recovering Missing Pixels for Landsat ETM+ SLC-off Imagery Using Multi-Temporal Regression Analysis and a Regularization Method. Remote Sensing of Environment, 131, 182-194.

[3] Zheng, Z.Z., Fan, D.M. and Yang, W.N. (2007) Application of 3S Integrated Tech- 
nology in Land Use Dynamic Monitoring. Journal of Southwest Jiaotong University, 42, 409-413.

[4] Tanm, M.Y., Lim, C., Lim, H. and Zhang, L.J. (2010) Study on Spatial and Temporal Characteristics of Urban Heat Island in Shenzhen Based on Landsat/TM. The 27 th Annual Meeting of the China Meteorological Association of Urban Meteorogy.

[5] Zhang, E.J., Zhao, X.Y. and Zhang, J.J. (2007) Research on Climate Change in Shenzhen in Recent 50 Years. Journal of Peking University (Natural Science Edition), 43, 535-541.

[6] Ge, W.Q., Zhou, H.M. and Yang, Y.M. (2006) Study on the Mitigation Effect of Urban Green Space on Heat Island Based on Remote Sensing and GIS. Remote Sensing Technology and Application, 21, 433-435.

[7] Van De Griend, A. and Owe, M. (1993) On the Relationship between Thermal Emissivity and the Normalized Difference Vegetation Index for Nature Surfaces International. Journal of Remote Sensing, 14, 1119-1131. https://doi.org/10.1080/01431169308904400

[8] Tan, M.H., Li, X.B. and Jun, L.X. (2003) The Driving Force of Urban Land Expansion in China. Economic Geography, 23, 635-639.

[9] Li, W.M., Xing, Q., Dong, K. and Zhang, X.M. (2016) Analysis of Land Use Change in Shijiazhuang Based on Landsat Image. Western Resources, 6, 176-179.

[10] Liu, J.Y., Liu, M.L. and Zhuang, D.F. (2002) Spatial Pattern Analysis of Recent Land Use Change in China. China Science: Geosciences, 32, 1031-1040. 